\title{
Evaluación de la eficacia y seguridad clínica del metoprolol en el infarto agudo de miocardio
}

\author{
En los pacientes con infarto agudo de miocardio, el tratamiento precoz \\ con metoprolol no disminuye la mortalidad, disminuye el riesgo de reinfarto \\ y de fibrilación ventricular pero aumenta el riesgo de shock cardiogénico.
}

In patients with acute myocardial infarction, early treatment with metoprolol does not decrease mortality, decreases the risk of reinfarction and ventricular fibrillation but increases the risk of cardiogenic shock.

Artículo: Chen ZM, Pan HC, Chen YP, Peto R, Collins $\mathrm{R}$, Jiang LX, et al, COMMIT (ClOpidogrel and Metoprolol in Myocardial Infarction Trial) collaborative group. Early intravenous then oral metoprolol in 45,852 patients with acute myocardial infarction: randomized placebocontrolled trial. Lancet. 2005;366:1622-32.

Antecedentes: el tratamiento a largo plazo con bloqueadores beta después de un infarto agudo de miocardio reduce el riesgo de muerte, de modo que por cada 42 pacientes tratados durante dos años se evita una muerte ${ }^{1}$. En las guías de práctica clínica tanto de American College of Cardiology/American Heart Association como de la Sociedad Europea de Cardiología, el tratamiento oral con bloqueadores beta en los pacientes con infarto de miocardio que no presentan contraindicación para su uso, es una recomendación de clase I con un nivel A de evidencia ${ }^{2,3}$.

No existen datos concluyentes sobre la eficacia del tratamiento intravenoso con bloqueadores beta en la fase aguda del infarto de miocardio ${ }^{2}$. En el estudio ISIS-1, la mortalidad en el día 7 de los pacientes con infarto agudo de miocardio tratados en las primeras 12 horas del comienzo de los síntomas con atenolol, inicialmente por vía intravenosa y posteriormente oral, fue $4,3 \%$ frente a $3,7 \%$ en los pacientes del grupo control (6 vidas salvadas por cada 1.000 pacientes tratados $)^{4}$. El estudio MIAMI no demostró una reducción significativa de la mortalidad a los 15 días en los pacientes tratados con metoprolol por vía intravenosa y luego oral $(4,3 \%$ frente a $4,9 \%$ en el grupo tratado con placebo $)^{5}$.

En los pacientes con infarto agudo de miocardio que son tratados con fibrinolíticos, el estudio TIMI-II demostró que el tratamiento precoz con metoprolol por vía intravenosa comparado con el tratamiento diferido con metoprolol por vía oral reducía el riesgo de reinfarto pero no la mortalidad ${ }^{6}$. Por último, un análisis post-hoc del estudio GUSTO 1 que analizó la mortalidad a los 30 días de los pacientes tratados con atenolol o no tratados con atenolol, mostró mayor mortalidad en los que recibieron atenolol vía intravenosa y oral cuando se compararon con los que únicamente recibieron tratamiento oral con atenolol $(3,1 \%$ en el grupo tratado con atenolol vía intravenosa y oral frente a $2,7 \%$ en el grupo tratado sólo con atenolol por vía oral $)^{7}$.

Objetivo: analizar el balance entre los riesgos y los beneficios del tratamiento precoz con metoprolol, primero por vía intravenosa y luego oral, en los pacientes con infarto agudo de miocardio con elevación del segmento ST.

Diseño, lugar, período de estudio: están descritos en el resumen del artículo anterior.

Pacientes (fig. 1): Los criterios de inclusión y criterios de exclusión y las características de los pacientes están descritos en el resumen del artículo anterior. 


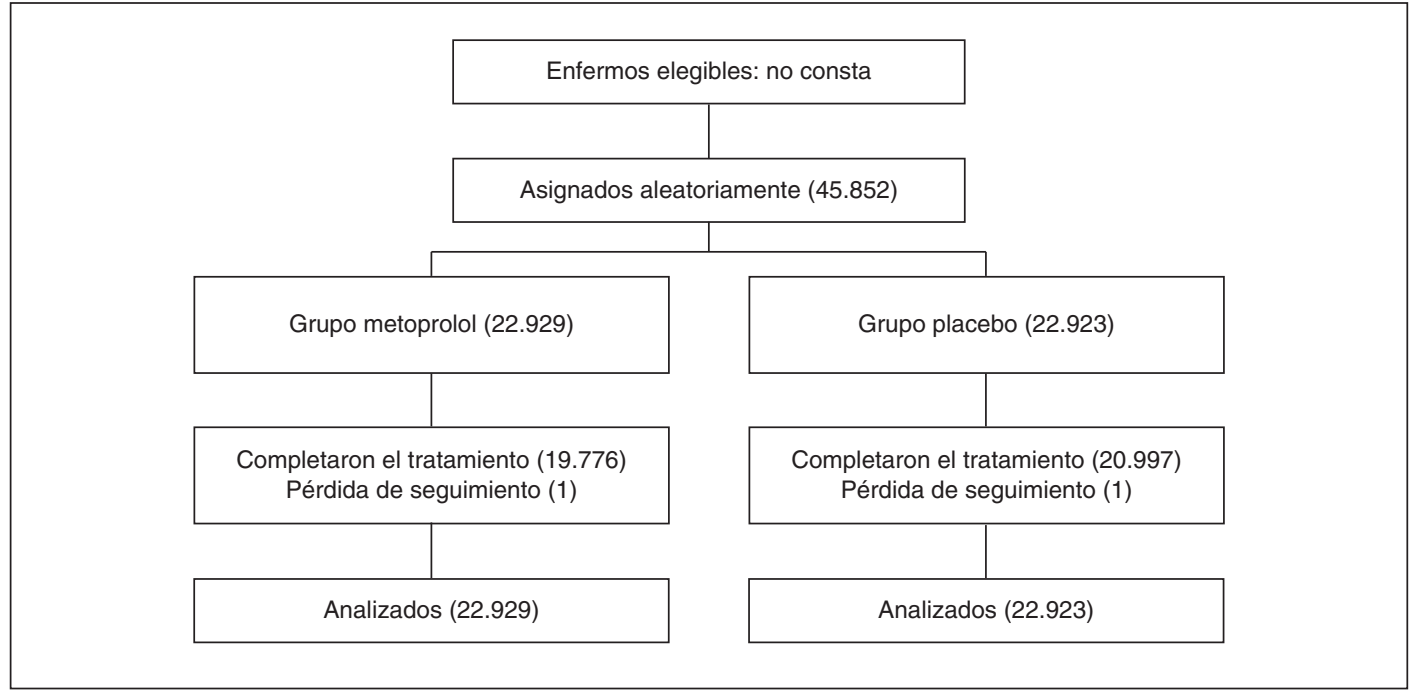

A diferencia de los ensayos clínicos previos, la insuficiencia cardíaca de clase Killip II o Killip III no se consideró un criterio de exclusión.

Intervenciones que se comparan: Grupo tratamiento con metoprolol: $5 \mathrm{mg}$ de metoprolol administrados en 2-3 minutos por vía intravenosa. Si la frecuencia cardíaca era superior a $50 \mathrm{lpm}$ y la tensión arterial sistólica permanecía por encima de $90 \mathrm{mmHg}$, se repetía la dosis de $5 \mathrm{mg}$ hasta en dos ocasiones, separada 2-3 minutos de la dosis previa. Transcurridos 15 minutos desde la administración intravenosa se comenzaba el tratamiento oral con $50 \mathrm{mg}$ cada 6 horas el primer día, y $200 \mathrm{mg} /$ día desde el segundo día hasta 4 semanas de tratamiento, o antes si el paciente moría o era dado de alta del hospital.

Grupo tratamiento con placebo: pauta similar a la anterior, pero con placebo.
El resto del tratamiento se dejó a discreción del médico responsable quien debía evitar el empleo de bloqueadores beta diferentes del metoprolol y de agentes antiplaquetarios distintos del ácido acetilsalicílico y el clopidogrel durante el período de estudio, a no ser que existiera una indicación establecida para ello.

Asignación aleatoria: sí. Oculta.

Enmascaramiento: sí.

Desenlaces principales: 1. desenlace combinado de muerte, reinfarto o parada cardíaca; 2 . muerte por cualquier causa durante los primeros 28 días o hasta el alta hospitalaria si se producía antes.

\section{Análisis por intención de tratar: sí.}

Resultados principales (tablas 1 y 2): la frecuencia de shock cardiogénico fue

Tabla 1: Desenlaces principales en los primeros 28 días o hasta el alta hospitalaria si se producía antes

\begin{tabular}{|l|c|c|c|c|}
\hline & Mortalidad & Reinfarto & $\begin{array}{c}\text { Parada cardíaca incluyendo } \\
\text { fibrilación ventricular }\end{array}$ & Desenlace combinado \\
\hline Grupo metoprolol & $1.774 / 22.929(7,7 \%)$ & $464 / 22.929(2,0 \%)$ & $1266 / 22.929(5,5 \%)$ & $2.166 / 22.929(9,4 \%)$ \\
Grupo placebo & $1.797 / 22.923(7,8 \%)$ & $568 / 22.923(2,5 \%)$ & $1.330 / 22.923(5,8 \%)$ & $2.261 / 22.923(9,9 \%)$ \\
RR (IC 95\%) & $1,01(0,95$ a 1,08$)$ & $0,82(0,72$ a 0,92$)$ & $0,95(0,88$ a 1,03$)$ & $0,96(0,91$ a 1,01$)$ \\
RAR (IC 95\%) & $0,1 \%(-0,4 \%$ a $0,6 \%)$ & $0,5 \%(0,2 \%$ a $0,7 \%)$ & $0,3 \%(-0,7 \%$ a $0,1 \%)$ & $0,4 \%(-0,1 \%$ a $1 \%)$ \\
RRR (IC 95\%) & $1,3 \%(-4,9 \%$ a $7,9 \%)$ & $18,3 \%(7,8 \%$ a $27,7 \%)$ & $4,8 \%(-2,6 \%$ a $11,6 \%)$ & $4,2 \%(-1,3 \%$ a $9,4 \%)$ \\
NNB (IC 95\%) & NA & NA $221(143$ a 500$)$ & NA & NA \\
\hline
\end{tabular}

NA: no aplicable.

IC: intervalo de confianza. 
Tabla 2: Efectos adversos ocurridos durante el tratamiento en el hospital

\begin{tabular}{|l|c|c|}
\hline & $\begin{array}{c}\text { Grupo metoprolol } \\
\mathrm{n}=22.929\end{array}$ & $\begin{array}{c}\text { Grupo placebo } \\
\mathrm{n}=22.923\end{array}$ \\
\hline $\begin{array}{l}\text { Insuficiencia cardíaca } \\
\quad \text { sin shock }\end{array}$ & $3.224(14,1 \%)$ & $2.902(12,7 \%)$ \\
Hipotensión persistente & $1.374(6,0 \%)$ & $668(2,9 \%)$ \\
Bradicardia & $1.235(5,4 \%)$ & $500(2,2 \%)$ \\
Ictus & $247(1,1 \%)$ & $220(1,0 \%)$ \\
$\begin{array}{l}\text { Rotura cardíaca } \\
\text { Tromboembolismo }\end{array}$ & $200(0,9 \%)$ & $233(1,0 \%)$ \\
$\quad$ pulmonar & $30(0,1 \%)$ & $35(0,2 \%)$ \\
\hline
\end{tabular}

significativamente superior en el grupo de metoprolol $(5,0 \%$ frente a 3,9\%, RR 1,29; intervalo de confianza al 95\%: 1,18 a 1,40; número necesario para perjudicar: 90 (IC 95\%: 67 a 143).

Información sobre costes: no consta.

Financiación del estudio y conflicto de intereses: figuran en el resumen anterior.

Conclusiones de los autores: el tratamiento precoz con bloqueadores beta en los pacientes con infarto agudo de miocardio reduce el riesgo de reinfarto y de fibrilación ventricular, pero aumenta el riesgo de shock cardiogénico, especialmente durante el primer día de evolución. Por lo tanto, la actitud más prudente sería considerar el tratamiento con bloqueadores beta únicamente cuando el paciente con infarto de miocardio se encuentre hemodinámicamente normal.

Conclusiones de los revisores: las conclusiones de los autores están en consonancia con los resultados del estudio. Después de años de incertidumbre sobre el efecto de los bloqeuadores beta administrados precozmente en la fase aguda del infarto de miocardio, un estudio metodológicamente adecuado y de gran tamaño muestral pone de manifiesto que el metoprolol por vía intravenosa y luego oral no tiene un efecto reductor de la mortalidad, aunque sí sobre la incidencia de reinfarto y de arritmias graves.

Los análisis post hoc realizados muestran ciertos hallazgos que permiten sentar nuevas hipótesis, que podrán ser contrastadas en el futuro, y que actualmente pueden orientar sobre el uso de metoprolol precozmente en los enfermos con infarto agudo de miocardio. El riesgo de shock cardiogénico con el uso de metoprolol se observa principalmente durante el primer día, y en los subgrupos de enfermos con: 1 . edad $\geq 70$ años, 2 . tensión arterial sistólica $<120 \mathrm{mmHg}$; 3. frecuencia cardíaca $>110$ lpm; 4. Killip III. Por el contrario, la reducción de la incidencia de reinfarto o arritmias graves aparece en el grupo tratado con metoprolol tras el primer día. Por tanto, parece juicioso reservar la administración de bloqueadores beta a los enfermos que muestren estabilidad hemodinámica tras 24 horas del comienzo de los síntomas de infarto agudo de miocardio, salvo que existan otras indicaciones para su uso.

\section{Bibliografía}

1. Freemantle N, Cleland J, Young P, Mason J, Harrison J. $B$ Blockade after myocardial infarction: systematic review and meta regression analysis. BMJ. 1998;318:1730-7.

2. Antman EM, Anbe DT, Armstrong PW, Bates ER, Green LA, Hand M, et al. ACC/AHA guidelines for the management of patients with ST-elevation myocardial infarction. Circulation. 2004;110:e82-292.

3. López-Sendón J, Swedberg K, McMurray J, Tamargo J, Maggioni AP, Dargie H, et al. Task ForceOn Beta-Blockers $\mathrm{f}$ the European Society of Cardiology. Expert consensus document on beta-adrenergic receptor blockers. Eur Heart J. 2004;25:1341-62.

4. ISIS-1 (First International Study of Infarct Survival) Collaborative Group. Randomised trial of intravenous atenolol among 16027 cases of suspected acute myocardial infarction: ISIS-1. Lancet. 1986;2:57-66.
5. The MIAMI Trial Research Group: metoprolol in acute myocardial infarction (MIAMI). A randomized placebo controlled international trial. Eur Heart J. 1985;6:199-211.

6. Roberts R, Rogers WJ, Mueller HS, Lambrew CT, Diver DJ, Smith HC, et al. Immediate versus deferred beta-blockade following thrombolytic therapy in patients with acute myocardial infarction: results of the Thrombolysis in Myocardial Infarction (TIMI) II-B Study. Circulation. 1991;83:422-37.

7. Pfisterer M, Cox JL, Granger CB, Brener SJ, Naylor CD, Califf RM, et al. Atenolol use and clinical outcomes after thrombolysis for acute myocardial infarction: the GUSTO-I experience. Global Utilization of Streptokinase and TPA (alteplase) for Occluded Coronary Arteries. J Am Coll Cardiol. 1998;32:634-40. 


\section{Glosario}

RR (riesgo relativo): es el cociente entre el riesgo de que ocurra un suceso en el grupo tratado dividido por el riesgo de que ocurra el suceso en el grupo control (habitualmente placebo).

RRR (reducción relativa del riesgo): es la reducción proporcional en el riesgo de que ocurra un suceso (por ejemplo, mortalidad) expresada en porcentaje. Se calcula $(1-\mathrm{RR} 1) * 100 \%$
RAR (reducción absoluta del riesgo): es la diferencia entre el riesgo de que ocurra un hecho suceso en el grupo control menos el riesgo en el grupo tratado expresada en porcentaje. Se calcula $\left(\mathrm{R}_{\mathrm{c}}-\mathrm{R}_{\mathrm{t}}\right) * 100$

NNB (número necesario para beneficiar): es el número de pacientes que hay que tratar para producir un efecto beneficioso $[\mathrm{NNB}=100 / \mathrm{RAR}]$. 